\title{
SPLINE FUNCTION APPROXIMATIONS FOR SOLUTIONS OF ORDINARY DIFFERENTIAL EQUATIONS
}

BY FRANK R. LOSCALZO ${ }^{1}$ AND THOMAS D. TALBOT

Communicated by Creighton Buck, January 4, 1967

1. Introduction and description of method. The known discrete variable methods for the solutions of differential equations (see [1]) furnish the approximate solutions as discrete tabular values at usually equidistant values of the independent variable. The object of this paper is to search for approximate solutions in the form of a spline function $S(x),[4]$, of degree $m(m \geqq 2)$ and class $C^{m-1}$. This approach was suggested to the authors by I. J. Schoenberg [3].

Let the differential equation be

$$
y^{\prime}=f(x, y), \quad 0 \leqq x \leqq b,
$$

about which we assume the following. If $T=\{(x, y) \mid 0 \leqq x \leqq b\}$, then we assume that $f(x, y) \in C^{m-2}$ in $T$ and that it satisfies the Lipschitz condition

$$
\left|f(x, y)-f\left(x, y^{*}\right)\right| \leqq L\left|y-y^{*}\right| \quad \text { if } 0 \leqq x \leqq b .
$$

If $m \geqq 3$ then (2) is equivalent to the boundedness of $\partial f / \partial y$ in $T$.

Our construction of the approximate solution $S(x)=S_{m}(x)$ is as follows. Let $y(x)$ be the solution of (1) determined by the initial value $y(0)=y_{0}$. Let $n$ be an integer $>m, h=b / n$ and let $S(x)(0 \leqq x \leqq b)$ be a spline function of degree $m$, class $C^{m-1}$ and having its knots at the points $x=h, 2 h, \cdots,(n-1) h$.

We define the first component of $S(x)=S_{m}(x)$ by

$$
\begin{aligned}
S(x)=y(0)+y^{\prime}(0) x+\cdots & +\frac{1}{(m-1) !} y^{(m-1)}(0) x^{m-1} \\
& +\frac{1}{m !} a_{0} x^{m} \quad(0 \leqq x \leqq h)
\end{aligned}
$$

with the last coefficient $a_{0}$ as yet undetermined. We now determine $a_{0}$ by requiring that $S(x)$ should satisfy (1) for $x=h$. This gives the equation

$$
S^{\prime}(h)=f(h, S(h))
$$

${ }^{1}$ Supported in part by the National Science Foundation under Grant GP-6070. 
to be solved for $a_{0}$. In terms of $\xi=a_{0} h^{m-1}(1-m)$ ! it is seen that $(4)$ is an equation in $\xi$ which is conveniently solved by iteration.

The polynomial (3) being determined, repeat the same steps in the interval $[h, 2 h]$ : we define

$$
S(x)=\sum_{\nu=0}^{m-1} \frac{1}{\nu !} S^{(\nu)}(h)(x-h)^{\nu}+\frac{1}{m !} a_{1}(x-h)^{m} \quad(h \leqq x \leqq 2 h)
$$

and determine $a_{1}$ such as to satisfy the equation

$$
S^{\prime}(2 h)=f(2 h, S(2 h)) \text {. }
$$

Continuing in like manner we evidently obtain a spline function $S_{m}(x)$ satisfying the equation

$$
S_{m}^{\prime}(\nu h)=f\left(\nu h, S_{m}(\nu h)\right) \quad(\nu=0,1, \cdots, n) .
$$

Theorem 1. If $h<m / L$ then the spline function $S_{m}(x)$ exists and is uniquely defined by the above construction.

2. The consistency relation for a spline function. Let $S$ denote the class of spline functions in $[0, b]$, of degree $m$, class $C^{m-1}$ with knots $x_{\nu}=\nu h(\nu=1, \cdots, n-1)$. Let $s(x) \in \mathcal{S}$. If restricted to the interval $[0,(m-1) h], s(x)$ depends on $(m+1)+(m-2)=2 m-1$ linear parameters. It follows that the $2 m$ quantities,

$$
s(\nu h), s^{\prime}(\nu h) \quad(\nu=0,1, \cdots, m-1),
$$

cannot be linearly independent. In fact there is a unique linear relation between them (Schoenberg [3]), namely

$$
\sum_{\nu=0}^{m-1} a_{\nu}^{(m)} s(\nu h)=h \sum_{\nu=0}^{m-1} b_{\nu}^{(m)} s^{\prime}(\nu h)
$$

whose coefficients may be described as follows: writing

$$
Q_{m}(x)=\frac{1}{(m-1) !} \sum_{i=0}^{m}(-1)^{i}\left(\begin{array}{c}
m \\
i
\end{array}\right)(x-i)_{+}^{m-1},
$$

which is a $B$-spline (see [4] and [5]), we have

$$
\begin{aligned}
& a_{\nu}^{(m)}=(m-1) !\left(Q_{m}(\nu)-Q_{m}(\nu+1)\right), \\
& b_{\nu}^{(m)}=(m-1) ! Q_{m+1}(\nu+1) .
\end{aligned}
$$

From (8) and (9) we easily obtain the numerical values of Table 1. 


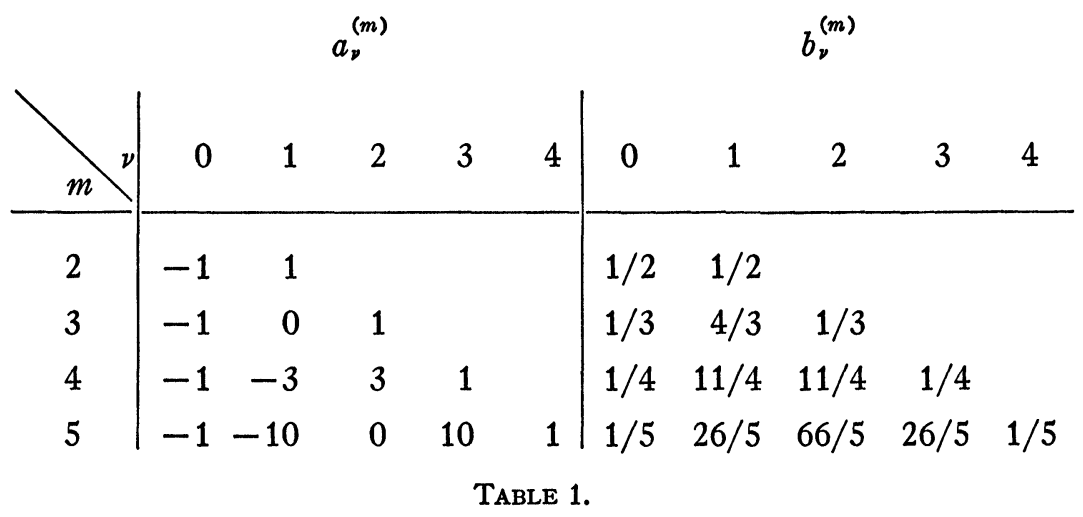

TheOREM 2. The values $S_{m}(\nu h)(\nu=0,1, \cdots, n)$ obtained in $\$ 1$ are precisely the values furnished by the discrete multistep method described by the recurrence relation

(10) $\sum_{\nu=0}^{m-1} a_{\nu}^{(m)} y_{k-m+1+\nu}=h \sum_{\nu=0}^{m-1} b_{\nu}^{(m)} y_{k-m+1+\nu}^{\prime} \quad(k=m-1, \cdots, n)$

if we use the starting values

$$
y_{0}=S_{m}(0), \quad y_{1}=S_{m}(h), \cdots, y_{m-2}=S_{m}((m-2) h) .
$$

Notice that our integration method, which appears as a one-step method in $\$ 1$, according to Theorem 2 furnishes also some of the solutions of the $(m-1)$-step method (10).

3. Quadratic spline functions and the trapezoidal rule. If we consult Table 1 and apply Theorem 2 for $m=2$, we find the corresponding 1-step method to be

$$
y_{k}-y_{k-1}=(h / 2)\left(y_{k-1}^{\prime}+y_{k}^{\prime}\right)=(h / 2)\left(f\left(x_{k-1}, y_{k-1}\right)+f\left(x_{k}, y_{k}\right)\right),
$$

which is the trapezoidal rule. Thus by Theorem 2 the trapezoidal rule will give the same discrete solution $S_{2}(\nu h)$ as the quadratic spline method described in $\$ 1$ for $m=2$.

Theorem 3. If $f(x, y) \in C^{2}$ in $T$, then a constant $K$ can be determined such that for all $h<2 / L$

$$
\begin{gathered}
\left|S_{2}(x)-y(x)\right|<K h^{2}, \quad\left|S_{2}^{\prime}(x)-y^{\prime}(x)\right|<K h^{2}, \\
\left|S_{2}^{\prime \prime}(x)-y^{\prime \prime}(x)\right|<K h,
\end{gathered}
$$


if $x \in[0, b]$, provided we define the value of the step function $S_{2}^{\prime \prime}(x)$ at a knot $x_{\nu}$ by the usual arithmetic mean.

4. Cubic spline functions and the Milne-Simpson method. Again from Table 1 we find for $m=3$ the recurrence relation

$$
\begin{aligned}
y_{k}-y_{k-2} & =(h / 3)\left(y_{k-2}^{\prime}+4 y_{k-1}^{\prime}+y_{k}^{\prime}\right) \\
& =(h / 3)\left(f\left(x_{k-2}, y_{k-2}\right)+4 f\left(x_{k-1}, y_{k-1}\right)+f\left(x_{k}, y_{k}\right)\right)
\end{aligned}
$$

which is one way of writing Simpson's rule. Hence, by Theorem 2, the Milne-Simpson method will furnish the discrete solution $S_{3}(\nu h)$, provided that we use as starting values $y_{0}$ and $y_{1}=S_{3}(h)$.

Theorem 4. If $f(x, y) \in C^{3}$ in $T$, then a constant $K$ can be determined such that for all $h<3 / L$

$$
\begin{aligned}
\left|S_{3}(x)-y(x)\right| & <K h^{4}, & \left|S_{3}^{\prime}(x)-y^{\prime}(x)\right| & <K h^{3}, \\
\left|S_{3}^{\prime \prime}(x)-y^{\prime \prime}(x)\right| & <K h^{2}, & \left|S_{3}^{\prime \prime}(x)-y^{\prime \prime \prime}(x)\right| & <K h
\end{aligned}
$$

if $x \in[0, b]$, provided we define the value of the step function $S_{3}^{\prime \prime \prime}(x)$ at a knot $x_{\nu}$ by the usual arithmetic mean.

An advantage of our procedure of $\S 1$ over the use of the recurrence relation (10) is this: the step size $h$ can be changed at each step, if necessary, without added complications. This remark adds flexibility to our version $(\$ 1, m=3)$ of the Milne-Simpson method.

\section{Higher degree spline functions and unstable multistep methods.}

Theorem 5. The solutions $S_{m}(x)$ are unstable as $h \rightarrow 0$ for $m \geqq 4$.

We may apply known criteria for multistep methods (Dahlquist's theorem; see [1, Theorem 5.5, p. 218]) to the relation (10) in order to judge the stability of our spline approximation $S_{m}(x)$. From (9) and (10) it appears that the stability depends on the zeros of the "associated polynomial"

$$
\sum_{\nu=0}^{m-1} a_{\nu}^{(m)} x^{\nu}=(x-1)(m-1) ! \sum_{\nu=0}^{m-2} Q_{m}(\nu+1) x^{\nu}
$$

Using (8) and (12) and the fact that the sum of the roots of a polynomial $p(x)=a_{0}+\cdots+a_{n-1} x^{n-1}+a_{n} x^{n}$ is $-a_{n-1} / a_{n}$, it is easily seen that the associated polynomial (12) has at least one root of modulus greater than unity for $m \geqq 4$. Therefore $S_{m}(x)$ is related by (10) to an unstable multistep method. Numerical results, obtained on the CDC 1604 at the University of Wisconsin Computing Center, for the equation $y^{\prime}=y, y(0)=1$ for $m=4,5,6,7$, show strong divergence. 
Acknowledgments. The authors are grateful to Professors Ramon E. Moore and I. J. Schoenberg for their valuable suggestions and encouragement in the preparation of this work.

\section{REFERENCES}

1. P. Henrici, Discrete variable methods in ordinary differential equations, Wiley, New York, 1962.

2. R. E. Moore, The automatic analysis and control of error in digital computation based on the use of interval numbers, Error in Digital Computation, Vol. I, L. B. Rall (ed ), Wiley, New York, 1965, pp. 61-130.

3. I. J. Schoenberg, private communication.

4. - On spline functions, Mathematics Research Center Tech. Summary Report No. 625 (1966).

5. - Contributions to the problem of approximation of equidistant data by analytic functions, Quart. Appl. Math. 4 (1946), 45-99 and 112-141.

Computer Sciences Department, University of Wisconsin 